\title{
BMJ Open Strategies to support HIV literacy in the roll-out of pre-exposure prophylaxis in Scotland: findings from qualitative research with clinical and community practitioners
}

\author{
Ingrid Young $\left(\mathbb{D},,^{1}\right.$ George Valiotis ${ }^{2}$
}

To cite: Young I, Valiotis G. Strategies to support HIV literacy in the roll-out of pre-exposure prophylaxis in Scotland: findings from qualitative research with clinical and community practitioners. BMJ Open 2020;10:e033849. doi:10.1136/ bmjopen-2019-033849

- Prepublication history for this paper is available online. To view these files, please visit the journal online (http://dx.doi org/10.1136/bmjopen-2019033849).

Received 26 August 2019 Revised 13 February 2020 Accepted 25 March 2020

\section{Check for updates}

(C) Author(s) (or their employer(s)) 2020. Re-use permitted under CC BY-NC. No commercial re-use. See rights and permissions. Published by BMJ.

${ }^{1}$ Centre for Biomedicine, Self and Society, Usher Institute, University of Edinburgh,

Edinburgh, UK

${ }^{2}$ European Health Management Association, Brussels, Belgium

Correspondence to

Dr Ingrid Young;

ingrid.young@ed.ac.uk

\section{ABSTRACT}

Objectives Limited understanding of pre-exposure prophylaxis (PrEP), coupled with negative public discourse, are significant barriers to its introduction. What works to support PrEP awareness and broader HIV literacy among diverse communities in the context of biomedical HIV prevention remains unclear. This article considers how PrEP can be translated across diverse communities and what the HIV literacy challenges might be in the current context of PrEP provision.

Setting We developed an HIV literacy informed community tool to support the roll-out of PrEP in Scotland. We undertook qualitative research with practitioners in urban and rural settings across nine Scottish health boards.

Primary outcome measure To examine HIV literacy challenges in the context of PrEP provision.

Participants Interviews and focus groups with community $(n=19)$ and clinical $(n=13)$ practitioners working with gay and bisexual men and African communities were undertaken between March and October 2017 concerning PrEP support, stigma and HIV literacy.

Results HIV literacy in the context of PrEP needs to consider more than the provision of individually targeted information. Practitioners identified and responded to stratified communities and social norms of knowledge, which influenced messaging, support and informed how practitioners enabled PrEP engagement and dialogue. Social barriers in HIV literacy, including structural stigmas relating to HIV and homophobia, shaped practitioner concerns and support for community members' willingness to engage with PrEP.

Conclusion Critical HIV literacy in the age of PrEP is a complex social practice. Attention needs to be paid to how information is provided and facilitates engagement, rather than simply what information is given.By exploring practitioner use of the Know about PrEP tool, we have shown how consideration of the patterns of access to services and information, the delivery of and support for engagement with PrEP information and the wider strategies employed to negotiate ongoing structural social barriers can support more equitable and diverse PrEP community conversations.
Strengths and limitations of this study

- This article reports on qualitative research with clinical and community practitioners who work with gay and bisexual men and men and women from African communities at the start of National Health Service (NHS) pre-exposure prophylaxis (PrEP) provision in Scotland.

- This article offers a theoretically informed approach to HIV literacy in the context of new biomedical HIV interventions, where there is a paucity of published research and reflection on HIV literacy challenges.

- The study recruited urban and rural participants who work across 9 of the 14 Scottish Health Boards to explore community and clinical components of the pathways to PrEP across Scotland.

- This article does not include data from PrEP users; further research exploring how potential and actual PrEP users engage with HIV information is warranted.

\section{INTRODUCTION}

Pre-exposure prophylaxis (PrEP) was made available through National Health Service (NHS) sexual health services in Scotland in July 2017. ${ }^{1}$ Scotland was the first country in the UK, and one of the first internationally, to prescribe PrEP through its national health services. However, questions remain as to how this new HIV prevention intervention can be effectively and equitably translated within and across highly diverse communities most affected by HIV, ${ }^{23}$ particularly for both gay and bisexual men and African men and women living in Scotland ${ }^{4}$ who are most likely to be considered candidates for PrEP in this setting. The socially complex challenges of access, uptake and sustained use have been well documented, ${ }^{5-7}$ as has increasing-if uneven-PrEP awareness and acceptability (primarily among gay and bisexual men) in the $\mathrm{UK}^{8-10}$ and elsewhere. ${ }^{11} 12$ Increasingly, 
PrEP research is paying attention to who knows about PrEP and how they come to learn about and trust it and how this is enabled. ${ }^{1314}$ As such, limited awareness and understanding of PrEP, along with a negative or stigmatising environment, can be seen as significant social barriers to the effective introduction of PrEP information for all communities affected by HIV.

Mainstream UK PrEP media coverage, especially in the run up to Scottish PrEP provision, framed PrEP as an intervention primarily for gay and bisexual men and focused largely on 'irresponsible' sexual practice of PrEP users, the high costs of PrEP and the well-rehearsed narrative of 'irresponsible' use of public funds for 'lifestyle choices'. ${ }^{15-17}$ This created a contentious environment within which PrEP information could be shared and discussed. Community and activist organisations in the UK have worked hard to counter negative media coverage and limited awareness through health promotion mechanisms such as websites, social media platforms, leaflets and engagement activities. ${ }^{18-20}$ While an emphasis has been placed on providing PrEP information around access, efficacy, use and dosing to potential PrEP users, what works to improve PrEP awareness and broader HIV literacy among significantly distinctive communities in the context of increasingly biomedicalised HIV prevention remains unclear. ${ }^{21}$ What is critical HIV literacy and how can it be mobilised for heterogenous PrEP users and wider communities affected by HIV? This article considers the use of a community HIV literacy tool designed explicitly to support PrEP engagement across heterogeneous communities affected by HIV and which was used as part of the roll-out of PrEP in Scotland to understand and address these issues. We ask: how can the translation of PrEP be supported within and across diverse communities and what are the HIV literacy challenges-and potential responses-in the current context of PrEP provision?

\section{DEVELOPING HIV LITERACY}

As many years of HIV education, health promotion and social research have shown, simply providing HIV information is not enough to improve HIV knowledge or the means to act on this information. ${ }^{22}{ }^{23}$ Increasingly, attention has been paid to health literacy and its role in shaping health practices both in and outside the clinic. ${ }^{24}$ Informed by Freire's critical pedagogy, ${ }^{25}$ some health literacy scholars argue that 'critical health literacy is, like community development, a process in which citizens become aware of issues, participate in critical dialogue, and become involved in decision making for health'. ${ }^{26}$ As such, critical health literacy is a complex 'social practice ${ }^{27}$ where people work together in networks, 'referencing common values and cultural practices to make sense of texts in order to achieve social goals ${ }^{28}$ Supporting critical health literacy, then, requires consideration of much more than increasing individual health knowledge. It means encouraging and enabling dialogue within and across groups, communities and institutions so that health-related information can be adapted and incorporated into social norms of health practices effectively and sustainably. ${ }^{7}$

While health literacy has received increasing attention in recent years, ${ }^{16}$ there has been less focus on HIV and sexual health literacy. ${ }^{29-31}$ This is especially important given the increasing role of biomedical HIV prevention and its role in sustainable contemporary HIV prevention efforts. Parker $e t a l^{2}$ argue that 'little has been done to articulate the pedagogical approaches through which knowledge about these existing tools might be best transmitted - let alone how decision might be made about which tools to use ${ }^{2}$, (p. 2). The Developing HIV Literacy Framework is a theoretically informed approach to critical HIV literacy, developed collaboratively with research, clinical and HIV community stakeholders in response to these issues and in response to the increasing role of biomedical prevention interventions such as PrEP. ${ }^{21}$ Here, HIV literacy is defined as:

The ability and skills of individuals and communities that means they are equipped and willing to engage with HIV, have access to and understand HIV information, able to apply learned HIV information within their sexual practice and able to engage with others about this HIV knowledge and related sexual practices. $^{21}$ (p. 1)

This approach establishes HIV literacy not as a knowledge-deficit model but rather as a complex social practice that is shaped by sexual partners, communities and the wider environment at each stage of identifying, taking up and using HIV information. ${ }^{27} 28$

Drawing on the Developing HIV Literacy Framework, ${ }^{21}$ we developed the Know about PrEP tool (figure 1) in late 2016 in anticipation of, but uncertainty around, Scottish PrEP provision. Negative media coverage, uncertain regulation, lack of PrEP promotion campaigns and ongoing HIV stigma were identified as significant initial barriers to: (1) willingness to engage with PrEP and (2) understandings of PrEP among people who might be potential PrEP users as well as wider publics. ${ }^{32}$ These issues would shape effective PrEP implementation in Scotland. Grounded in a critical HIV literacy approach, this community HIV literacy tool was designed to introduce PrEP at the peer level through supported community conversations. The tool was the use of a multifold business card sized leaflet with minimal text (maximum 15 words per panel) and visual images, designed to answer common questions about PrEP and intended to support PrEP conversations. It was not tailored to specific communities affected by HIV nor was not designed to comprehensively answer all PrEP questions. Instead, it was designed to answer and encourage discussion around a set of questions (see figure 1) identified through the authors engagements with community stakeholders, participation in, and observation of, PrEP media interviews and as part of their professional work. The tool was intended to provide support for and enable community and health practitioners-who may or may 


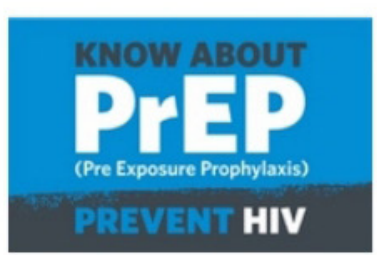

Introductory Panel

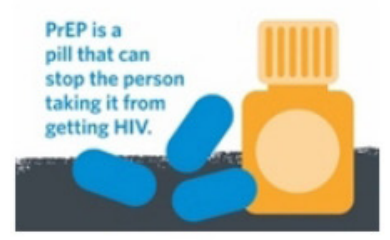

What is PrEP?

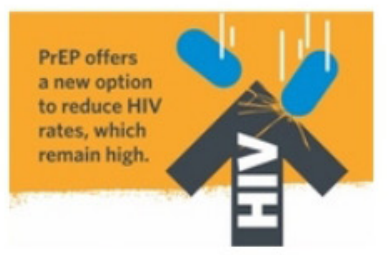

Why do we need

PrEP?

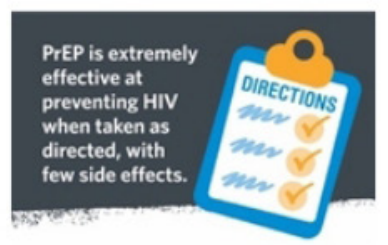

Does it work?
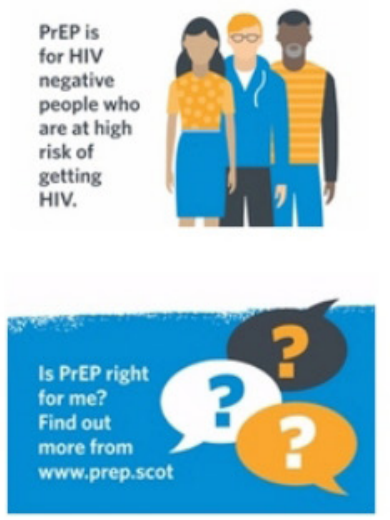

Who is it for?

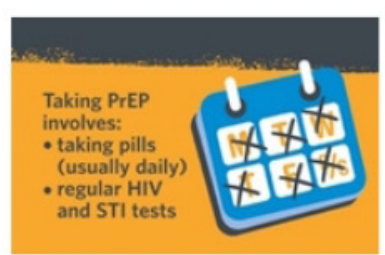

What is involved in

taking PrEP?

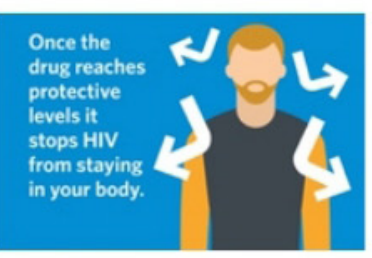

How does it work?

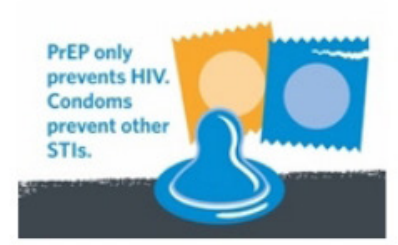

What about

condoms?

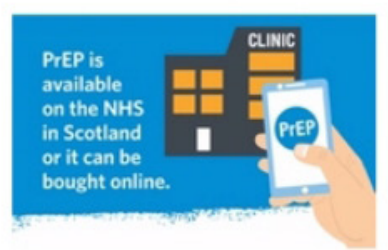

Where can I get

PrEP?

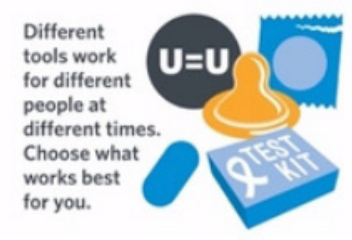

How does PrEP work

with other HIV

prevention tools?

\section{Who made this \\ leaflet?}

Figure 1 Know about PrEP tool: supporting community conversations. The figure presents an image of the Know about PrEP tool. The tool is the use of a multifold business card sized leaflet, featuring 12 separate panels with a maximum of 15 words per panel and visual images which reflect PrEP messaging. Each full colour panel responds to a singular question that sits next to the image in the figure. The panel messaging is meant to provide answers to commonly asked questions in a non-stigmatising way, and the use of this tool is meant to support informal PrEP conversations. PrEP, pre-exposure prophylaxis.

not be PrEP experts themselves-to address common and sometimes challenging questions in the context of PrEP stigma ${ }^{32}$ and to be adapted to specific communities and diverse settings by the practitioners using it. The tool was designed by IY, GV and Kelsey Smith (HIV Scotland), reflecting migrant, lesbian, gay, bisexal (LGB) and cis-gendered perspectives. Additionally, we sought input from Developing HIV Literacy group comprised of community members and patients, as well as clinical and community practitioners and academics, including those who work with and/or identify as gay and bisexual men and/or African men and women. ${ }^{21}$

After a pilot period with community organisations between March and May 2017, the Know about PrEP tool was made available in June 2017 (see box 1) to community and clinical organisations who worked with communities affected by HIV and who might benefit from PrEP. In the Scottish context, gay and bisexual men, and Black African communities living in Scotland are those most at risk of and affected by $\mathrm{HIV}^{4}$ and considered as the primary 
Box 1 Know about PrEP community tool development

Problem: lack of pre-exposure prophylaxis (PrEP) knowledge within communities affected by HIV, including potential PrEP users, their sexual partners, peers and wider networks.

Context: UK media coverage of PrEP in 2016 where homophobia, HIV and PrEP stigma hinders PrEP learning and discussions in public fora and community settings and between sexual partners.

Aims: support the introduction of PrEP to people and communities who knew very little about it within the context of increased PrEP stigma.

Method: tool to support PrEP conversations within community settings for use with and by PrEP users, sexual partners and peers.

Tool development timeline:

October-December 2016: tool concept and design, drawing on Developing HIV Literacy Framework; addressing 'willingness to engage' and 'understanding' of individuals, sexual partners and peers.

January-May 2017: tool piloted with community organisations who work with communities affected by HIV who might benefit from PrEP in urban and rural settings

March-May 2017: qualitative data collection with partner community organisations to collect feedback and understand use of the tool.

March-May 2017: informal feedback sought from National Health Service (NHS) clinicians and health practitioners involved in sexual health via email and in consultation with Developing HIV Literacy research partners.

June 2017: feedback and policy change incorporated into final version of tool.

July 2017: PrEP made available through Scottish NHS sexual health services.

June-0ctober 2017: tool distributed through community organisations who work with communities affected by HIV who might benefit from PrEP and NHS sexual health clinics in urban and rural settings across Scotland.

August-October 2017: qualitative data collection with community and clinical practitioners who provide support for or prescribe PrEP and who used the Know about PrEP tool across Scotland.

candidates for PrEP at the time of the study. The tool was distributed to and used by third sector HIV and sexual health organisations across Scotland as part of their health promotion and support work with these groups. It was also distributed through NHS health promotion networks and made available through sexual health services who prescribe PrEP in all 14 Scottish Health boards. The tool linked to a national website-prep.scot—which provided digital images of the leaflet and included information about how to access PrEP through NHS clinics.

\section{METHODS}

To understand with whom and how the tool worked and to better understand HIV literacy challenges in the context of PrEP provision, IY undertook a mixture of qualitative interviews and focus groups with clinical and community practitioners, and community volunteers. These methods were selected in order to allow for an in-depth and wideranging exploration of HIV literacy issues in relation to and beyond the use of the tool, as well as flexibility to accommodate practitioner availability. As such, individual or group interviews or focus groups were offered. ${ }^{33}{ }^{34}$ We undertook

\begin{tabular}{|c|c|c|}
\hline $\begin{array}{l}\text { Sexual and } \\
\text { gender identity }\end{array}$ & $\begin{array}{l}17 \\
\text { heterosexual } \\
\text { cis women }\end{array}$ & $\begin{array}{l}15 \text { Gay and bisexual cis } \\
\text { men }\end{array}$ \\
\hline Ethnicity & $\begin{array}{l}25 \text { white } \\
\text { participants }\end{array}$ & $\begin{array}{l}7 \text { participants of colour } \\
\text { including black African, } \\
\text { black Caribbean and } \\
\text { South Asian }\end{array}$ \\
\hline Living with HIV & $\begin{array}{l}5 \text { participants } \\
\text { living with HIV }\end{array}$ & $\begin{array}{l}27 \text { participants HIV } \\
\text { negative or did not } \\
\text { disclose their status }\end{array}$ \\
\hline \multirow[t]{4}{*}{$\begin{array}{l}\text { Professional role } \\
\text { and community } \\
\text { of focus }\end{array}$} & \multirow{4}{*}{$\begin{array}{l}13 \text { clinical } \\
\text { practitioners, } \\
\text { including HIV } \\
\text { clinicians, } \\
\text { sexual health } \\
\text { nurses and } \\
\text { healthcare } \\
\text { assistants }\end{array}$} & $\begin{array}{l}19 \text { community health } \\
\text { promotion practitioners } \\
\text { and/or volunteers, of } \\
\text { whom: }\end{array}$ \\
\hline & & $\begin{array}{l}6 \text { work with black African } \\
\text { men and women }\end{array}$ \\
\hline & & $\begin{array}{l}8 \text { work with gay and } \\
\text { bisexual men }\end{array}$ \\
\hline & & $\begin{array}{l}5 \text { work across both } \\
\text { communities }\end{array}$ \\
\hline
\end{tabular}

purposive sampling with community and clinical HIV practitioners who provided and/or supported PrEP in some way, had been provided with the Know about PrEP tool and were using it as part of their work. Community support for PrEP at the time of the research was primarily limited to gay and bisexual men and, to a lesser extent,African men and women; organisations working with or comprised of trans communities, people engaged in sex work and/ or people injecting drugs were not knowingly engaged in (or funded for) PrEP support at the time of this study. Participants were invited to take part in the study via emails sent through existing health promotion networks in NHS boards across urban and rural Scotland to reach clinical practitioners and through known third sector organisations who work with specified communities. Efforts were made to ensure inclusion of participants who work with-and where possible, self-identify as-communities affected by HIV in Scotland. IY conducted five individual interviews $(n=5)$, six group interviews $(n=12)$ and three focus groups $(n=15)$ with community practitioners and/or community members $(n=19)$ and clinical practitioners $(n=13)$ involved in the roll-out of PrEP across Scotland (see table 1 for participant characteristics). Data collection took place between MarchOctober 2017; data collection was stopped when a sufficient number and diversity of participants was reached in relation to geographic diversity, clinical and community practice and when recruitment pathways were exhausted within the time period of the funded study. Participants included practitioners working with gay and bisexual men, African heterosexual men and women and black and minority ethnic (BME) gay men across urban and rural Scotland, and one participant from outside of Scotland. Participants reflect a wide range of intersectional identities and experiences in relation to gender, sexuality, race, class, disability 
and geographic location. The majority of community practitioners identified as members of the communities with whom they worked. IY, who undertook all the data collection, has worked in collaboration with community and clinical partners in the Scottish sexual health sector since 2011 through research, policy and community engagement events. Although IY is not from either community in question nor is a clinical or community practitioner, she sought to pay attention and attend to any power imbalances in data collection process through well-established reflexive practices. ${ }^{35}$ During the interviews and focus groups, participants were asked to reflect on: what PrEP information was asked about or discussed; their use of the leaflet; their wider PrEP discussions; and their observations around stigma and other PrEP barriers. Participants were provided with a study information sheet, and written consent was secured from all participants at the start of each interview and focus group.All data were audio-recorded, transcribed, checked for quality and accuracy and anonymised. In order to look across community and clinical practitioner experiences, as well as to consider issues across sexuality, gender and race, data from interviews and focus groups were analysed together. Analysis was an iterative process: IY undertook a preliminary inductive analysis of the data to identify key themes by reading and rereading the transcripts. This preliminary analysis was then explored by IY and GV with wider Developing HIV Literacy project academic, clinical and community partners ${ }^{21}$ at a half-day workshop, where we identified, explored and probed key themes through group discussions. IY then undertook systematic data analysis to identify and expand key themes presented in this article ${ }^{33}$ with the aid of NVivo V.10 qualitative data analysis software. Sense checking of the themes and coded data was done throughout the analysis with GV and in collaboration with another qualitative researcher, Nicola Boydell, who assisted on the Developing HIV Literacy project. We identified three broad themes in relation to critical HIV literacy, which we explore below. Illustrative extracts are presented in box 2 .

\section{Patient and public involvement}

The Developing HIV Literacy group was involved in the codesign of the Developing HIV Literacy Framework, provided feedback on the design and modifications of the Know about PrEP tool and contributed to the design and undertaking of the qualitative research reported on in this study. The Developing HIV Literacy group was comprised of community members and patients, as well as community and clinical practitioners and academics who met over the course of the 3-year project. ${ }^{21}$

\section{RESULTS}

\section{Stratified communities and social norms of knowledge}

We found that practitioners identified and responded to stratified communities and social norms of PrEPand HIV-knowledge. This patterning of social norms of PrEP knowledge shaped what practitioners felt was appropriate messaging and support, and guided if and how they used the tool to help facilitate PrEP conversations. For instance, practitioners noted the importance of presenting the right amount of information to introduce PrEP and described how the tool was especially helpful to use with people who had no or little PrEP knowledge. The simplicity and clarity of the tool and the succinct information it presented was often contrasted with other available PrEP material, such as the i-base Guide to PrEP in the UK. ${ }^{18}$ The highly comprehensive i-base leaflet was described as more appropriate for those with advanced knowledge of PrEP (extract 1A). Participants working in a clinical context identified people newly diagnosed with HIV as candidates for the tool and described it as a potential resource to support discussions with these patients about HIV prevention with their sexual partners until their viral loads became undetectable (extract 1B).

However, within NHS services, clinical practitioners reported predominantly gay and bisexual men accessing PrEP services, who they assessed as having significant PrEP knowledge and high HIV literacy more generally. As a result of these patterns of clinic attendance for PrEP, clinical practitioners saw the tool as less helpful in supporting conversations with already well-informed patients attending for PrEP and highlighted their need for more 'advanced' information (extract 1C). Clinical practitioners identified how the social norms of PrEP knowledge among the majority of attending patients meant that they sought materials that contained more clinical information around dosing and required testing, rather than those that supported general PrEP conversations. This is suggestive of who was accessing PrEP services in the early phase of implementation in Scotland and how PrEP awareness and HIV literacy among service users in these clinical settings might be stratified by access to services and the social norms of knowledge across attending patients.

\section{Enabling engagement and dialogue}

In contrast to clinical practitioners who predominantly used the tool to provide information for individual patients, community practitioners reported using the tool as the means through which they could enable and encourage interpersonal and community level discussions about PrEP and related HIV prevention issues, facilitating both engagement with and dialogue about PrEP. In particular, community practitioners and volunteers working with gay and bisexual men explained how the tool helped to structure PrEP conversations undertaken during community outreach and health promotion activities by providing a guide or prompts in relation to specific PrEP issues and concerns (extract 2A). Others described 'creative' ways of using and sharing the tool to engage others, raising awareness of PrEP through the physical use of the tool and not simply relying on PrEP information it contained (extract 2B). Community practitioners also described how the tool facilitated the introduction of PrEP-related information and discussions, such as risk 


\section{Box 2 Illustrative extracts}

1. Stratified communities and social norms of knowledge

A. 'It's very straight to the point, it's facts, it's informative as opposed to screeds and screeds of information that sometimes people don't need, they just need, like, that is enough' (Community practitioner with gay and bisexual men).

B. 'I've had one woman who we diagnosed as HIV-positive and she was worried about her relationship and wanted to be sexually active and things. I talked about PrEP for her just at least until she gets her viral load down to an undetectable level for her partner, so I gave her that information and the leaflet 'cause she genuinely knew nothing about PrEP, so in that sense the leaflet was useful for her and her partner' (Clinical practitioner).

a. 'Most of the men I think that l've seen could tell me about PrEP... basically they're so well informed when they come into the service, they've obviously done a lot of background research and are well aware of how it works and also the criteria for eligibility and things as well, they're very much aware of that, they've all looked it up online and things. I mean, I do tend to give this leaflet out but I think that most of the men, from how I see it, have already gone (and) surpassed the information that's on the leaflet' (Clinical practitioner).

2. Enabling engagement and dialogue

A. '... [Y]ou opened it up and basically talk about each section as you go down, so you'll go, "this is PrEP, this is what it is, it's a tablet blah, blah, it prevents this, you take it..." so yeah, just going through literally pointing out each section and then answering any questions that you get... it was interesting to kind of point to a section and go, "this is the literature and that, this section we're talking about" and then they can focus on the question... it just flowed nicely' (Community practitioner with gay and bisexual men).

B. 'I actually use the leaflet as a tool cause I had a lanyard and what I done is I stuck it basically to the name badge, so it said "let's talk about PrEP" and people are going "what's that?" cause it was literally on my chest and they're looking at it, so I used it as a tool itself' (Community practitioner with gay and bisexual men).

C. ' ... start talking to them about what it is and what it's for, and in that situation at those health fairs l've been met with what seems to be some interest, but then you move onto the effects of the drug itself, how the drug works and then I start talking about Can't Pass it On and at that point it seems to resonate with them' (Community practitioner with gay and bisexual men).

D. 'There's a group in [the local] University that took a big handful for their little groups to give that out, the guy that took them obviously knew all about PrEP and stuff and was "oh we need that to kind of educate people", so there's kind of... "I know what it is, I want to education others"' (Community practitioner with gay and bisexual men).

3. Decodifying social barriers to HIV literacy

A. 'It's the issue that's always existed... once I associate with anything to do with HIV there's [the] implication that I am positive myself, so engaging with the intro as it is I think there needs to be a layer that comes a bit more, I think it's more like the testing campaign that [is taking place] this week where we have two strap lines, one of them is about one in 20 Africans living with HIV is living with HIV in Scotland, do you know your status? But the other one is about we have tested 250 people, we have helped 250 people, we know their status, do you know yours? And that's about coming from that other side where someone can engage with it and say "oh okay other people have tested, I can go", rather than "oh so one in 20 "' (Community practitioner with African communities).

B. 'No I personally felt that, maybe it's because of what my job is I know quite a lot about this, I personally and I'm talking as a professional not as a lay person, I personally was reading it and I thought this is talking to gay men because no young African person would consider themselves at risk after reading this' (Community practitioner with African communities).

C. 'I think for the science-y stuff, you know, presenting stuff in Proud or IPERGAY or the evidence around dosing, right, that just needs to be presented as plain scientific fact. I think it's the stuff around understanding the barriers to why people might not take up PrEP or why they might not perceive risk a certain way; that then needs to be couched in culturally specific terms.... very often people say "so you're having that dirty weekend in Berlin" right okay, whereas I go "well Femmie, your girlfriend Adioka is coming in from Nigeria for the weekend" that makes it culturally specific' (Community practitioner with both African communities and gay and bisexual men).

D. 'I'm looking for gay men or men who would identify as just having sex with other men type thing. But the majority of the time you've got other people standing at the table as well who are like, I don't know, somebody who's like a typical plumber type person who's maybe standing beside somebody who's maybe, say for example, this is stereotyping, like a hairdressing student and the hairdressing student doesn't want to turn round and say something in front of the plumber, you know ... in case he gets outed by everybody else, you know, because he's still scared of the stigma that he got at school. Cause you leave school, you go to college, you reinvent yourself' (Community practitioner with gay and bisexual men).

E. 'I think if somebody wants to come and talk to me about it, they've got the opportunity because if my gaydar's working alright ... I give out that wee bit more extra information to somebody, but at the same time trying to make it look as if I'm giving out other stuff to other people, you know, but being careful with my leaflets in what l'm giving to what, you know. Or if there's somebody there, like for example a group of girls and one of them will say "oh my pal he's gay" and l'm like "oh tell him about this, tell him about that" "oh I never knew you could get things like that?" cause they're quite good as well, doing a wee bit of peer education' (Community practitioner with gay and bisexual men).

assessment, testing, condom use and treatment as prevention ( $\mathrm{U}=\mathrm{U}$ ) (extract 2C), allowing PrEP discussions to be situated within wider social norms of community safer sex practices. The tool was seen as helpful to engage with potential PrEP users and for use by community volunteers and community members to discuss PrEP with their sexual partners, peers and family (extract 2D). In this way, the tool was seen to support discussions among sexual partners and peers by individuals who were already deemed to be highly HIV literate but who were not health practitioners themselves.

\section{Decodifying social barriers to HIV literacy}

Practitioners described how identifying and responding to social barriers in HIV literacy, including structural stigmas relating to HIV and homophobia, ${ }^{36}$ shaped their concerns about and support for community members' willingness to engage with PrEP. In particular, community practitioners 
described negotiating —or decodifying ${ }^{25}$ — these social barriers and reflected on if, how and where the PrEP tool was supportive.

Practitioners working with African communities explained how the provision of simple PrEP information could not address all barriers to engaging in PrEP conversations. One participant explained how HIV stigma is a major barrier to peoples' willingness to engage with HIVrelated information and emphasised the need to pay attention to implicit assumptions about, and wording around, 'normative' health practices such as regular HIV testing (extract 3A). Moreover, practitioners highlighted how words intended to be neutral may still implicitly speak to a specific group-and not to another-because of assumptions about social context and engagement with health issues. For instance, one community practitioner suggested that the wording used in the tool- 'for people at high risk'-may not speak to African (or BME) communities because of community perceptions of low HIV risk and safer sex practices, in spite of supporting epidemiological evidence (extract 3B). Community practitioners-especially those working with African communities-outlined how engaging with community members was reliant on more than simply presenting key information but delivering the information in ways that were socially and/or culturally specific to different audiences. For instance, this meant recognising how and when commonly used PrEP scenarios that often accompany PrEP information could be adapted to relevant social norms and contexts (extract 3C). Practitioners worked with the tool to tailor their community engagement, rather than only relying only on the printed material.

While many of the clinical practitioners in this study described gay and bisexual men attending clinics for PrEP as having good HIV literacy, community practitioners highlighted further social barriers among this heterogeneous community. For example, community practitioners explained the ways in which homophobia continued to play a key role in how gay and bisexual men, especially younger men and those outside of larger urban centres, were negotiating their sexual identities in diverse social settings. In the context of perceived or potential homophobia, community practitioners described how they would discretely use the tool at sexual health promotion events to provide information or contacts when face-to-face conversations were not possible and emphasised the need to maintain discretion in these settings (extract 3D). Community practitioners also tailored their messages and materials to people with whom they engaged and used the tool to encourage and support peer education where they could not directly engage with gay and bisexual men (extract $3 \mathrm{E})$.

\section{DISCUSSION}

This article has demonstrated that HIV literacy in the context of PrEP needs to consider more than simply the provision of information targeted at the individual level;
HIV literacy is shaped by stratified communities and social norms of knowledge, enabled through engagement and dialogue and negotiated in the context of wider social barriers such as HIV stigma and homophobia. By exploring practitioner use of the Know about PrEP tool, we have shown how consideration of the patterns of access to services and information, the delivery of and support for engagement with PrEP information and the wider strategies employed to negotiate ongoing structural social barriers can support more equitable and diverse PrEP community conversations. Our findings have important implications for HIV literacy in relation to PrEP and also for the wider implementation of and support for new HIV pharmaceutical interventions that directly consider the impact of existing social inequalities and diverse communities affected by HIV in Scotland and the UK more generally. ${ }^{2}$

We have shown how assumptions about social norms around risk and sexual health may be built into what is incorrectly assumed to be neutral language and messaging and which may inadvertently close down engagement with those already marginalised, such as African communities and/or those with limited HIV knowledge. However,the provision of what is seen as clinical information can be shaped to appropriately engage with specific social norms and community practices. Moreover, we identified how structural stigmas around HIV and homophobia played a role in if and how this information could be shared, and where community practitioners drew on strategies to overcome or respond to specific social barriers to enable engagement. Our findings, therefore, offer insight into HIV literacy as a complex social practice ${ }^{27}$ shaped by social norms of knowledge and stratified within and across communities. An apparent 'unwillingness' to engage with PrEP may not always reflect a lack of knowledge but be grounded in structural stigmas and where PrEP information may play a part in compounding existing inequalities. These findings echo those of a recent study that has shown how community norms could act as a barrier to PrEP knowledge, discussions and use among black gay and bisexual men in the UK. ${ }^{9}$ That diverse communities beyond white, urban, cis-gendered gay and bisexual men are not accessing PrEP ${ }^{1}$ may be, in part, due a lack of support in navigating complex social knowledge networks and stigmas to access services.

Our work also highlights diverse HIV literacy needs of communities affected by HIV as identified by clinical and community practitioners and how these are patterned by setting, social norms and community practices. There is a need to introduce appropriately tailored HIV literacyinformed PrEP interventions that address existing inequalities in PrEP awareness and access. While there is a growing body of research on PrEP communications and how trust is built with potential PrEP users in relation to effectiveness, ${ }^{13}$ our work illustrates how PrEP promotion and support strategies need to consider more than messaging; PrEP support needs to engage with how HIV literacies are shaped by structural factors forces such as 
geography, community norms of knowledge and access to services and how these may be patterned by sexuality, gender and/or race/ethnicity. ${ }^{12}$ Current UK PrEP guidelines ${ }^{37}$ outline non-clinical and structural factors that may indicate PrEP need among communities with limited access to PrEP (eg, trans communities and women) and go some way to addressing these issues. However, Calabrese $e t a l^{8}$ show how clinical eligibility criteria itself can still exclude women as potential PrEP candidates by focusing on epidemiological risk factors associated with gay and bisexual men. Calabrese et al show how this can directly result in limiting PrEP awareness at a community level and, ultimately, risks deprioritising PrEP for women at a policy level. Our findings, therefore, suggest that a failure to address the diverse HIV literacy needs of stratified communities could have significant implications for PrEP access and support policy for already underserved communities.

Finally, our research suggests attention needs to be paid to how information is provided and facilitates engagement, rather than simply what information is given. Practitioners judged the community tool to be suited to people who knew very little about PrEP in the context of supporting non-clinical discussions. This was because it was understood to enable informed yet informal discussions with and between sexual partners and peers. The community-focused approach of the tool could go some way to supporting more meaningful engagement with PrEP in the context of social barriers where there is widespread recognition that more needs to be done to expand awareness beyond people already accessing PrEP. Although advocating community-based approaches to HIV prevention may not be a 'new' idea, ${ }^{22}$ attention needs to be paid to reanimating this approach for HIV literacy interventions; HIV literacy interventions need to consider and respond to community social and sexual practices in the context of PrEP and recognise how 'communitybased expertise is crucial to inform and shape... pedagogical approaches' ${ }^{2}$ (p.7) to HIV prevention. Indeed, PrEP organisations in the UK such as PrEPster are currently undertaking peer-led education programmes with women from black African, Caribbean and trans and non-binary communities as a key strategy to address existing PrEP inequalities. ${ }^{39}$

\section{Strengths and limitations}

This paper offers timely insight into PrEP literacy and awareness in both an urban and rural context at the start of PrEP provision in one of the first European countries to make it available. We offer a theoretically informed approach to HIV literacy in the context of new biomedical HIV interventions, of which there is a paucity of published research and reflection. Due to the timescale and scope of this project, we did not undertake research across all Scottish NHS boards. Clinical practitioners in other, smaller boards may reflect different experiences of this this tool. The aim of this research was to better understand if, how and where clinical and community practitioners could be supported in the introduction of PrEP, as such we did not undertake any research with community users of the PrEP tool. Further research exploring how potential and actual PrEP users engage with the information is warranted, as is more in-depth work with specific communities such as African men and women and transgender communities.

\section{CONCLUSIONS}

Our research has shown that supporting critical HIV literacy in the context of PrEP needs: more explicit understandings of how social norms of knowledge are stratified within and across communities; to consider strategies that enable engagement and dialogue with potential PrEP users and wider communities; and respond directly to structural stigmas that shape and perpetuate social inequalities. These findings illustrate the importance of theoretically informed HIV literacy tools to address complex structural barriers. Since the roll-out of PrEP, community organisations across the UK have adapted the tool for women ${ }^{40}$; created an expanded booklet to support to PrEP community champions ${ }^{41}$ and creating a similar tool for $\mathrm{U}=\mathrm{U}$ education. ${ }^{42}$ Working with communities to adapt these tools and approaches is essential. As Parker et al argue, this work needs to recognise 'existing expertise and a shifting of power to people and communities to make decisions regarding the HIV prevention options that best fit their lived realities'2 (p. 7). Such work will further add to our understanding of HIV literacy and how to translate PrEP within and across communities in a way that is accessible, supported beyond access to clinics and helps to reduce existing inequalities.

Acknowledgements The Know about PrEP community tool was jointly designed by IY, GV and Kelsey Smith. Thanks to all Developing HIV Literacy research, clinical and community collaborators who provided feedback on the Know About PrEP community tool. Thanks to Nicola Boydell, Martin Holt, Catherine Dodds and Paul Flowers for their helpful comments on the draft paper.

Contributors IY codesigned the Know about PrEP tool, designed and undertook the research, undertook the analysis and drafted the article; GV codesigned of the Know about PrEP tool and contributed to this article.

Funding This research was funded by Scottish Chief Scientist Office Postdoctoral Fellowship (PDF/14/02; CF/CSO/02). IY is currently a member of the Centre for Biomedicine, Self and Society, supported by Wellcome (209519/Z/17/Z). GV was CEO of HIV Scotland when the research was undertaken.

Competing interests None declared.

Patient consent for publication Not required.

Provenance and peer review Not commissioned; externally peer reviewed.

Data availability statement Data are available on reasonable request. Anonymised transcripts can be made available on request by contacting the lead author. Use of the data will be agreed in discussion with the lead author.

Open access This is an open access article distributed in accordance with the Creative Commons Attribution Non Commercial (CC BY-NC 4.0) license, which permits others to distribute, remix, adapt, build upon this work non-commercially, and license their derivative works on different terms, provided the original work is properly cited, appropriate credit is given, any changes made indicated, and the use is non-commercial. See: http://creativecommons.org/licenses/by-nc/4.0/.

ORCID iD

Ingrid Young http://orcid.org/0000-0002-1242-5992 


\section{REFERENCES}

1 ISD HPS. Implementation of HIV PrEP in Scotland: First Year Report. Health Protection Scotland, Information Services Division, 2019. Available: https://www.hps.scot.nhs.uk/web-resources-container/ implementation-of-hiv-prep-in-scotland-first-year-report/ [Accessed 16 Jun 2019].

2 Parker RG, Perez-Brumer A, Garcia J, et al. Prevention literacy: community-based advocacy for access and ownership of the HIV prevention toolkit. J Int AIDS Soc 2016;19:21092.

3 Newman C, Hughes S, Persson A, et al. Promoting 'Equitable Access' to PrEP in Australia: Taking Account of Stakeholder Perspectives. AIDS Behav 2019;23:1846-57.

4 Cullen B, Cameron R, Henderson D, et al. HIV treatment and care in Scotland: summary report to 31 December 2018. Glasgow: : Health Protection Scotland, 2019. Available: https://hpspubsrepo.blob.core. windows.net/hps-website/nss/2849/documents/1_hiv-in-scotlandsummary-2018.pdf

5 Keogh P, Dodds C. Pharmaceutical HIV prevention technologies in the UK: six domains for social science research. AIDS Care 2015;27:796-803.

6 Hannaford A, Lipshie-Williams M, Starrels $\mathrm{JL}$, et al. The use of online posts to identify barriers to and facilitators of HIV preexposure prophylaxis (PrEP) among men who have sex with men: a comparison to a systematic review of the peer-reviewed literature. AIDS Behav 2018;22:1080-95.

7 Kippax S, Stephenson N, Parker RG, et al. Between individual agency and structure in HIV prevention: understanding the middle ground of social practice. Am J Public Health 2013;103:1367-75.

8 Young I, Flowers P, McDaid LM. Barriers to uptake and use of preexposure prophylaxis (PrEP) among communities most affected by HIV in the UK: findings from a qualitative study in Scotland. BMJ Open 2014:4:e005717.

9 Witzel TC, Nutland W, Bourne A. What are the motivations and barriers to pre-exposure prophylaxis (PrEP) use among black men who have sex with men aged 18-45 in London? results from a qualitative study. Sex Transm Infect 2019;95:262-6.

10 Frankis J, McDaid L, Wallace L, et al. P447 Awareness and acceptability of pre-exposure prophylaxis among MSM: results from Scotland's gay bar survey. BMJ Sex Transm Infect;95:A201.

11 Lachowsky NJ, Lawson Tattersall T, Sereda P, et al. Community awareness of, use of and attitudes towards HIV pre-exposure prophylaxis (PrEP) among men who have sex with men in Vancouver, Canada: preparing health promotion for a publicly funded PrEP program. Sex Health 2019;16:180-6.

12 Morgan J, Ferlatte O, Salway T, et al. Awareness of, interest in, and willingness to pay for HIV pre-exposure prophylaxis among Canadian gay, bisexual, and other men who have sex with men. Can J Public Health 2018;109:791-9.

13 Underhill K, Morrow KM, Colleran C, et al. Explaining the efficacy of pre-exposure prophylaxis (PrEP) for HIV prevention: a qualitative study of message framing and messaging preferences among US men who have sex with men. AIDS Behav 2016;20:1514-26.

14 Collier KL, Colarossi LG, Sanders K. Raising awareness of pre-exposure prophylaxis (PrEP) among women in New York City: community and provider perspectives. J Health Commun 2017;22:183-9.

15 Megarry D. Journalism Union condemns "homophobic tropes" used in PrEP coverage - Gay Times., 2016. Available: https://www. gaytimes.co.uk/community/44856/journalism-union-condemnshomophobic-tropes-used-prep-coverage/ [Accessed 16 Jun 2019].

16 Twocock P. The Daily Mail's PrEP story was like going back to the dark old days of the 80s | Paul Twocock | Opinion | The Guardian,
2016. Available: https://www.theguardian.com/commentisfree/2016/ aug/04/daily-mail-prep-dark-old-days-80s-Igbt-hiv [Accessed 16 Jun 2019].

17 Mowlabocus S. 'What a skewed sense of values': Discussing PrEP in the British press. Sexualities 2019;17:136346071987272.

18 i-base. UK Guide to PrEP 1st edition [leaflet]. London, 2016.

19 Pebody R. PrEP Briefing paper, 2015. Available: http://www.aidsmap. com/PrEP/page/2983346 [Accessed 16 Jun 2019].

20 Feustel N. PrEP17 - The coming of age of PrEP. Gerogetown Media, 2017. Available: https://vimeo.com/214791866 [Accessed 16 Jun 2019].

21 Young I. Making the case for HIV literacy: a developing HIV literacy report. University of Edinburgh, 2019. Available: https://www. research.ed.ac.uk/portal/files/109716752/Making_the_Case_for_HIV Literacy.pdf

22 Kippax S, Race K. Sustaining safe practice: twenty years on. Soc Sci Med 2003;57:1-12.

23 Aggleton P, Yankah E, Crewe M. Education and HIV/AIDS-30 years on. AIDS Educ Prev 2011;23:495-507.

24 Nutbeam D, McGill B, Premkumar P. Improving health literacy in community populations: a review of progress. Health Promot Int 2018;33:901-11.

25 Freire P. Pedagogy of the Oppresed. London: Penguin, 1970.

26 Sykes S, Wills J, Rowlands G, et al. Understanding critical health literacy: a concept analysis. BMC Public Health 2013;13:150.

27 Samerski S. Health literacy as a social practice: social and empirical dimensions of knowledge on health and healthcare. Soc Sci Med 2019;226:1-8.

28 Chinn D. Critical health literacy: a review and critical analysis. Soc Sci Med 2011;73:60-7.

29 Gilbert M, Michelow W, Dulai J, et al. Provision of online HIV-related information to gay, bisexual and other men who have sex with men: a health literacy-informed critical appraisal of Canadian agency websites. Sex Health 2019;16:39-46.

30 Gilbert M, Dulai J, Wexel D, et al. Health literacy report. Available: http://www.ohtn.on.ca/healthliteracy/ [Accessed 16 Jun 2019].

31 McDaid L, Flowers P, Ferlatte O, et al. Sexual health literacy among gay, bisexual and other men who have sex with men: a conceptual framework for future research. Cult Health Sex 2020:1-17.

32 Golub SA. PrEP stigma: implicit and explicit drivers of disparity. Curr HIVIAIDS Rep 2018;15:190-7.

33 Mason J. Qualitative researching. London: Sage, 2017.

34 Barbour R. Doing focus groups. London: Sage, 2018.

35 Finlay L. Reflexivity: A Practical Guide for Researchers in Health and Social Science. Blackwell Publishing, 2003.

36 Parker R, Aggleton P. HIV and AIDS-related stigma and discrimination: a conceptual framework and implications for action. Soc Sci Med 2003;57:13-24.

37 Brady Met al. BHIVA/BASHH guidelines on the use of HIV preexposure prophylaxis (PrEP). London: British HIV Association; British Association of Sexual Health and HIV, 2018.

38 Calabrese SK, Willie TC, Galvao RW, et al. Current US guidelines for prescribing HIV pre-exposure prophylaxis (PrEP) Disqualify many women who are at risk and motivated to use PrEP. J Acquir Immune Defic Syndr 2019;81:395-405.

39 PrEPster. MobPrESH, 2019. Available: https://prepster.info/ mobpresh/ [Accessed 16 Jun 2019].

40 Sophia Forum. PrEP for Women [leaflet]. London: : i-base, 2017. Available: http://i-base.info/wp-content/uploads/2017/11/PrEPwomen-2017e.pdf

41 Developing HIV Literacy, HIV Scotland, PrEPster. Know about PrEP: Prevent HIV [booklet]. University of Edinburgh, 2017.

42 Waverley Care. $U=U$ [leaflet]. Edinburgh, 2018. 